\title{
Scheme for calculating the orbital-dependent exchange-correlation potential using the virial theorem: Application to atomic systems
}

\author{
M. Kodera, ${ }^{1, *}$ K. Higuchi, ${ }^{1}$ A. Narita, ${ }^{2}$ and M. Higuchi ${ }^{3}$ \\ ${ }^{1}$ Graduate School of Advanced Sciences of Matter, Hiroshima University, Higashi-Hiroshima 739-8530, Japan \\ ${ }^{2}$ Division of Applied Mathematics, Akita National College of Technology, Akita 011-8511, Japan \\ ${ }^{3}$ Department of Physics, Faculty of Science, Shinshu University, Matsumoto 390-8621, Japan
}

(Received 7 April 2008; published 2 July 2008)

\begin{abstract}
We present a density-functional scheme for calculating the orbital-dependent exchange-correlation potential using the virial theorem as a sum rule. In order to check the validity of this scheme, atomic-structure calculations only with the exchange potential are performed. The accuracy of this scheme is shown to be comparable to that of the optimized effective potential (OEP) method, while the computational workload is extremely reduced compared to the OEP method.
\end{abstract}

DOI: 10.1103/PhysRevA.78.012501

PACS number(s): 31.10.+z, 31.15.E-, 31.15.V-

\section{INTRODUCTION}

The local-density approximation (LDA) of the densityfunctional theory (DFT) $[1,2]$ has achieved considerable success in describing the ground-state electronic structure of solids in many cases. But, on the other hand, some defects of the LDA have been pointed out, e.g., the underestimation of the energy gap of semiconductors and insulators, underestimation of the exchange energy for atomic systems, and discrepancies of the equilibrium lattice constant and cohesive energy with experiments [3].

One of the promising methods beyond the LDA is the optimized effective potential (OEP) method [4-6]. In the OEP method, since the exchange-correlation energy functional is explicitly expressed by Kohn-Sham (KS) orbitals, many computational tasks are needed to solve the OEP integral equation that contains the exchange-correlation potential. Therefore, it is important to reduce the computational tasks of deriving the exchange-correlation potential when the OEP method is applied to solids.

Some approximations have been proposed to derive the exchange-correlation potential without solving the OEP integral equation directly [7-17]. Among them, the Krieger-LiIafrate (KLI) approximation is one of the most commonly used methods [7]. It is known that the KLI approximation gives a reasonable result to the OEP integral equation [7]. Although it actually reduces the computational workload fairly, the KLI approximation still needs heavy computational tasks if we apply it to solids.

In order to overcome this problem, we propose a method to derive the orbital-dependent exchange-correlation potential, which is more than just an approximation to the OEP integral equation. Our idea is quite simple. We start with the coupling-constant expression for the exchange-correlation energy functional. Our method is to derive the exchangecorrelation potential by utilizing the virial theorem [18] instead of taking the functional derivative of the paircorrelation function with respect to the electron density. Details will be shown in the next section.
The organization of this paper is as follows. In Sec. II, we present the theoretical framework of our method. In order to show not only the accuracy but also the calculation speed of our method, we perform the atomic-structure calculations from $\mathrm{He}$ to $\mathrm{Xe}$. The explicit expression for the exchangeonly calculation is given in Sec. III. The results and discussions are shown in Sec. IV. Finally, concluding remarks are given in Sec. V.

\section{THEORY}

The exchange-correlation energy functional of the DFT is expressed in terms of the exchange-correlation hole $\rho_{\mathrm{xc} \sigma}\left(\mathbf{r}, \mathbf{r}^{\prime}\right)$ as

$$
E_{\mathrm{xc}}[\rho]=\sum_{\sigma=\uparrow, \downarrow} \frac{e^{2}}{2} \iint d \mathbf{r} d \mathbf{r}^{\prime} \frac{\rho_{\sigma}(\mathbf{r}) \rho_{\mathrm{xc} \sigma}\left(\mathbf{r}, \mathbf{r}^{\prime}\right)}{\left|\mathbf{r}-\mathbf{r}^{\prime}\right|},
$$

with

$$
\rho_{\sigma}(\mathbf{r})=\sum_{i=1}^{N_{\sigma}}\left|\psi_{i \sigma}(\mathbf{r})\right|^{2}
$$

and

$$
\rho_{\mathrm{xc} \sigma}\left(\mathbf{r}, \mathbf{r}^{\prime}\right) \equiv \sum_{\sigma^{\prime}=\uparrow, \downarrow} \rho_{\sigma^{\prime}}(\mathbf{r})\left\{g_{\sigma \sigma^{\prime}}\left(\mathbf{r}, \mathbf{r}^{\prime}\right)-1\right\},
$$

where $g_{\sigma \sigma^{\prime}}\left(\mathbf{r}, \mathbf{r}^{\prime}\right)$ is the coupling-constant integrated paircorrelation function [3]. Then, the exchange-correlation potential is formally given by

$$
\begin{aligned}
V_{\mathrm{xc} \sigma}(\mathbf{r})= & \frac{\delta E_{\mathrm{xc}}[\rho(\mathbf{r})]}{\delta \rho_{\sigma}(\mathbf{r})} \\
= & e^{2} \int d \mathbf{r}^{\prime} \frac{\rho_{\mathrm{xc} \sigma}\left(\mathbf{r}, \mathbf{r}^{\prime}\right)}{\left|\mathbf{r}-\mathbf{r}^{\prime}\right|} \\
& +\frac{e^{2}}{2} \sum_{\sigma^{\prime}, \sigma^{\prime \prime}} \iint d \mathbf{r}^{\prime} d \mathbf{r}^{\prime \prime} \frac{\rho_{\sigma^{\prime}}\left(\mathbf{r}^{\prime}\right) \rho_{\sigma^{\prime \prime}}\left(\mathbf{r}^{\prime \prime}\right)}{\left|\mathbf{r}^{\prime}-\mathbf{r}^{\prime \prime}\right|} \frac{\delta g_{\sigma^{\prime} \sigma^{\prime \prime}}\left(\mathbf{r}^{\prime}, \mathbf{r}^{\prime \prime}\right)}{\delta \rho_{\sigma}(\mathbf{r})} .
\end{aligned}
$$

Our idea is to renormalize the second term of Eq. (4) into a

*mkodera@hiroshima-u.ac.jp 
constant factor $\beta$ multiplied by the first term. Namely, we suppose that the exchange-correlation potential is rewritten as

$$
V_{\mathrm{xc} \sigma}(\mathbf{r})=\beta e^{2} \int d \mathbf{r}^{\prime} \frac{\rho_{\mathrm{xc} \sigma}\left(\mathbf{r}, \mathbf{r}^{\prime}\right)}{\left|\mathbf{r}-\mathbf{r}^{\prime}\right|} .
$$

The soundness of this approximation may be confirmed by the fact that the first and second terms of Eq. (4) have similar spatial profiles except for their magnitudes $[19,20]$.

In order to determine the constant $\beta$, we utilize the virial theorem of the DFT [6]. It is given by

$$
T[\rho]-T_{s}[\rho]+E_{\mathrm{xc}}[\rho]+\sum_{\sigma} \int d \mathbf{r} \rho_{\sigma}(\mathbf{r}) \mathbf{r} \cdot \nabla V_{\mathrm{xc} \sigma}(\mathbf{r})=0,
$$

where $T[\rho]$ and $T_{s}[\rho]$ are the kinetic energy functionals for interacting and noninteracting systems, respectively. Substituting Eq. (5) into Eq. (6), we obtain

$$
\beta=\frac{T_{s}[\rho]-T[\rho]-E_{\mathrm{xc}}[\rho]}{\sum_{\sigma} \int d \mathbf{r} \rho_{\sigma}(\mathbf{r}) \mathbf{r} \cdot \nabla\left(e^{2} \int d \mathbf{r}^{\prime} \frac{\rho_{\mathrm{xc} \sigma}\left(\mathbf{r}, \mathbf{r}^{\prime}\right)}{\left|\mathbf{r}-\mathbf{r}^{\prime}\right|}\right)}
$$

If the explicit forms of the energy functionals, $E_{\mathrm{xc}}[\rho][$ or $\left.\rho_{\mathrm{xc} \sigma}\left(\mathbf{r}, \mathbf{r}^{\prime}\right)\right]$, and $T[\rho]-T_{s}[\rho]$ are given, then the exchangecorrelation potential can be obtained immediately from Eqs. (5) and (7). The orbital-dependent forms of $E_{\mathrm{xc}}[\rho]$, which have been usually used in the OEP method, would be among of the most promising ones beyond the LDA. Also concerning $T[\rho]-T_{s}[\rho]$, some approximations have been presented so far [3]. It should be noted that we do not have to solve the OEP integral equation even if we utilize the orbitaldependent expression for $E_{\mathrm{xc}}[\rho]$.

\section{EXCHANGE-ONLY CALCULATION}

For the purpose of checking the validity of the present scheme, we present in this section the expression for the exchange potential including the renormalization factor $\beta_{x}$. As usual, the Hartree-Fock (HF) expression is employed as the exchange energy. We have

$$
\begin{aligned}
E_{x}\left[\left\{\psi_{j \tau}\right\}\right]= & -\frac{e^{2}}{2} \sum_{\sigma=\uparrow, \downarrow} \sum_{j, k=1}^{N_{\sigma}} \iint d \mathbf{r} d \mathbf{r}^{\prime} \\
& \times \frac{\psi_{j \sigma}^{*}(\mathbf{r}) \psi_{k \sigma}^{*}\left(\mathbf{r}^{\prime}\right) \psi_{k \sigma}(\mathbf{r}) \psi_{j \sigma}\left(\mathbf{r}^{\prime}\right)}{\left|\mathbf{r}-\mathbf{r}^{\prime}\right|} .
\end{aligned}
$$

The exchange energy is also expressed by

$$
E_{x}[\rho]=\sum_{\sigma=\uparrow, \downarrow} \frac{e^{2}}{2} \iint d \mathbf{r} d \mathbf{r}^{\prime} \frac{\rho_{\sigma}(\mathbf{r}) \rho_{x \sigma}\left(\mathbf{r}, \mathbf{r}^{\prime}\right)}{\left|\mathbf{r}-\mathbf{r}^{\prime}\right|},
$$

where $\rho_{x \sigma}\left(\mathbf{r}, \mathbf{r}^{\prime}\right)$ is the coupling-constant integrated exchange hole. From Eqs. (8) and (9), $\rho_{x \sigma}\left(\mathbf{r}, \mathbf{r}^{\prime}\right)$ is given by

$$
\rho_{x \sigma}\left(\mathbf{r}, \mathbf{r}^{\prime}\right)=-\sum_{j, k=1}^{N_{\sigma}} \frac{\psi_{j \sigma}^{*}(\mathbf{r}) \psi_{k \sigma}^{*}\left(\mathbf{r}^{\prime}\right) \psi_{k \sigma}(\mathbf{r}) \psi_{j \sigma}\left(\mathbf{r}^{\prime}\right)}{\rho_{\sigma}(\mathbf{r})} .
$$

The virial relation (6) can be divided into two parts, i.e., exchange and correlation parts. They are, respectively, given by

$$
E_{x}[\rho]+\sum_{\sigma} \int d \mathbf{r} \rho_{\sigma}(\mathbf{r}) \mathbf{r} \cdot \nabla V_{x \sigma}(\mathbf{r})=0
$$

and

$$
T[\rho]-T_{s}[\rho]=-E_{c}[\rho]-\sum_{\sigma} \int d \mathbf{r} \rho_{\sigma}(\mathbf{r}) \mathbf{r} \cdot \nabla V_{c \sigma}(\mathbf{r}) .
$$

The exchange part can be derived by means of the technique of the electron-coordinate scaling [21], and the correlation part is defined as the difference between Eqs. (6) and (11). It should be noted that the kinetic energy functional $T[\rho]$ $-T_{s}[\rho]$ is contained only in the correlation part [22], i.e., we do not need to take into account the kinetic energy functional in the exchange-only (x-only) calculations. Thus, in the $\mathrm{x}$-only calculation, the renormalization factor $\beta_{x}$ is determined by Eq. (11). The exchange potential is expressed by

$$
V_{x \sigma}(\mathbf{r})=\beta_{x} e^{2} \int d \mathbf{r}^{\prime} \frac{\rho_{x \sigma}\left(\mathbf{r}, \mathbf{r}^{\prime}\right)}{\left|\mathbf{r}-\mathbf{r}^{\prime}\right|},
$$

where

$$
\beta_{x}=\frac{-E_{x}[\rho]}{\sum_{\sigma} \int d \mathbf{r} \rho_{\sigma}(\mathbf{r}) \mathbf{r} \cdot \nabla\left(e^{2} \int d \mathbf{r}^{\prime} \frac{\rho_{x \sigma}\left(\mathbf{r}, \mathbf{r}^{\prime}\right)}{\left|\mathbf{r}-\mathbf{r}^{\prime}\right|}\right)} .
$$

\section{RESULTS AND DISCUSSIONS}

In this section, calculation results of the present scheme are compared with those of various X-only calculations. We make comparisons with the $\mathrm{HF}$ as other works of the OEP method usually do. We shall show the advantage of the present scheme from the viewpoints of not only the accuracy but also the calculation speed.

\section{A. Exchange energy}

In Table I, we show the calculation results of the exchange energies for atoms from He to Ar, together with those of the HF [23], x-only local-spin-density approximation (xLSDA), OEP [24], and KLI approximation. The present scheme improves substantially upon the xLSDA, and reproduces the HF energy as accurately as the KLI approximation. The exchange energies of the present scheme are also in very good agreement with those of the OEP method.

In Fig. 1, we show the errors of the exchange energies for various $\mathrm{x}$-only calculations. The error is defined as

$$
\Delta_{\mathrm{ex}} \equiv\left|\frac{E-E_{\mathrm{HF}}}{E_{\mathrm{HF}}}\right| \text {, }
$$

where $E_{\mathrm{HF}}$ is the exchange energy of the HF, and where $E$ corresponds to the exchange energy of the xLSDA or KLI approximation or the present scheme. The errors of the 
TABLE I. Atomic exchange energies calculated from the HF, xLSDA, OEP, KLI, and present schemes. The HF and OEP results are taken from Refs. $[23,24]$, respectively. All values are in Rydberg units.

\begin{tabular}{lccccc}
\hline \hline & HF & xLSDA & OEP & KLI & Present \\
\hline $\mathrm{He}$ & -2.052 & -1.706 & -2.052 & -2.052 & -2.052 \\
$\mathrm{Li}$ & -3.562 & -3.009 & -3.562 & -3.562 & -3.571 \\
$\mathrm{Be}$ & -5.334 & -4.556 & -5.332 & -5.334 & -5.341 \\
$\mathrm{~B}$ & -7.488 & -6.460 & & -7.497 & -7.502 \\
$\mathrm{C}$ & -10.09 & -8.819 & & -10.10 & -10.10 \\
$\mathrm{~N}$ & -13.19 & -11.66 & -13.21 & -13.21 & -13.17 \\
$\mathrm{O}$ & -16.35 & -14.55 & & -16.37 & -16.32 \\
$\mathrm{~F}$ & -20.00 & -17.94 & & -20.01 & -19.94 \\
$\mathrm{Ne}$ & -24.22 & -21.87 & -24.21 & -24.20 & -24.09 \\
$\mathrm{Na}$ & -28.04 & -25.40 & -28.03 & -28.01 & -27.96 \\
$\mathrm{Mg}$ & -31.98 & -29.07 & -31.98 & -31.97 & -31.96 \\
$\mathrm{Al}$ & -36.14 & -32.91 & & -36.13 & -36.11 \\
$\mathrm{Si}$ & -40.56 & -37.02 & & -40.55 & -40.53 \\
$\mathrm{P}$ & -45.28 & -41.42 & -45.27 & -45.27 & -45.23 \\
$\mathrm{~S}$ & -50.00 & -45.68 & & -50.00 & -49.94 \\
$\mathrm{Cl}$ & -55.02 & -50.54 & & -55.02 & -54.96 \\
$\mathrm{Ar}$ & -60.38 & -55.55 & -60.35 & -60.35 & -60.28 \\
\hline \hline
\end{tabular}

present scheme and the KLI approximation are much smaller than those of the xLSDA. They are within $1 \%$. This is caused by the fact that the present scheme and the KLI approximation adopt the exchange energy functional that is free from the self-interaction.

\section{B. Total energy}

In Tables II-IV, we show the calculation results of the ground-state total energies for atoms from He to Xe, together with those of the HF [25], xLSDA, OEP [25], and KLI approximation. Similarly to the case of the exchange energy, the present scheme improves on the xLSDA, and reproduces

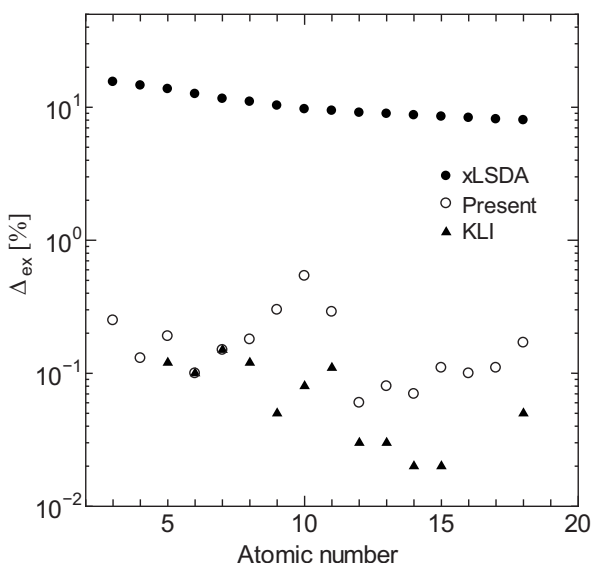

FIG. 1. Errors of the exchange energies of the present scheme are shown for atoms from $\mathrm{Li}$ to $\mathrm{Ar}$, together with those of the xLSDA and the KLI approximation. the HF results comparably to the OEP method and the KLI approximation.

\section{Calculation time}

In order to evaluate the computational workload of the present scheme, we compare the calculation times to be required for one iteration with those of the KLI approximation. In Fig. 2, we plot the results of the calculation times. As the atomic number increases, the difference of the calculation times between both methods becomes much larger. For example, for the $\mathrm{Ne}$ atom, the present scheme is about five times as fast as the KLI approximation. Also for the Xe atom, the present scheme is about 60 times as fast as the KLI approximation. The present scheme is proportional to the square of the atomic number, while the KLI approximation is proportional to the cube of the atomic number. Therefore, the calculation time of the present scheme is reduced by one order of magnitude in the atomic number.

As for the total calculation time of the self-consistent calculation, since the present scheme is easier to converge than the KLI approximation, the present scheme also takes much less time than the KLI approximation. For heavy atoms, the total calculation time of the present scheme is less than the calculation time to derive the exchange potential per iteration of the KLI approximation. Also from these results, the present scheme demands much less numerical work than the KLI approximation.

These improvements on the calculation speed come from the difference of the expressions for the exchange potentials. The KLI exchange potential is written as $[7,26]$

$$
\begin{aligned}
V_{x \sigma}^{\mathrm{KLI}}(\mathbf{r})= & \frac{e^{2}}{2 \rho_{\sigma}(\mathbf{r})} \sum_{i=1}^{N_{\sigma}}\left|\psi_{i \sigma}(\mathbf{r})\right|^{2}\left[u_{x i \sigma}(\mathbf{r})+u_{x i \sigma}^{*}(\mathbf{r})\right. \\
& \left.+2 \bar{V}_{x i \sigma}^{\mathrm{KLI}}-\bar{u}_{x i \sigma}-\bar{u}_{x i \sigma}^{*}\right],
\end{aligned}
$$

with

$$
\begin{gathered}
u_{x i \sigma}(\mathbf{r})=\frac{1}{\psi_{i \sigma}^{*}(\mathbf{r})} \frac{\delta E_{x}\left[\left\{\psi_{j \tau}\right\}\right]}{\delta \psi_{i \sigma}(\mathbf{r})}, \\
\bar{V}_{x i \sigma}^{\mathrm{KLI}}=\bar{V}_{x i \sigma}^{S}+\sum_{j=1}^{N_{\sigma^{-}}} M_{j i \sigma}\left\{\bar{V}_{x j \sigma}^{\mathrm{KLI}}-\frac{1}{2}\left(\bar{u}_{x j \sigma}+\bar{u}_{x j \sigma}^{*}\right)\right\}, \\
\bar{V}_{x i \sigma}^{S}=\int d \mathbf{r} \frac{\left|\psi_{i \sigma}(\mathbf{r})\right|^{2}}{2 \rho_{\sigma}(\mathbf{r})} \sum_{j=1}^{N_{\sigma}}\left|\psi_{j \sigma}(\mathbf{r})\right|^{2}\left[u_{x j \sigma}(\mathbf{r})+u_{x j \sigma}^{*}(\mathbf{r})\right],
\end{gathered}
$$

and

$$
M_{j i \sigma}=\int d \mathbf{r} \frac{\left|\psi_{j \sigma}(\mathbf{r})\right|^{2}\left|\psi_{i \sigma}(\mathbf{r})\right|^{2}}{\rho_{\sigma}(\mathbf{r})} .
$$

In Eq. (16), terms except the constant ones within the parentheses on the right-hand side can be expressed by using the exchange hole, such that 
TABLE II. Total energies for atoms from He to Ar, which are calculated from the HF, xLSDA, OEP, KLI, and present schemes. The HF and OEP total energies are taken from Ref. [25]. All values are in Rydberg units.

\begin{tabular}{lrrrrr}
\hline \hline & HF & xLSDA & OEP & KLI & Present \\
\hline $\mathrm{He}$ & $-5.7234^{\mathrm{a}}$ & -5.4473 & $-5.7234^{\mathrm{a}}$ & -5.7233 & -5.7234 \\
$\mathrm{Li}$ & -14.8655 & -14.3868 & -14.8650 & -14.8648 & -14.8572 \\
$\mathrm{Be}$ & -29.1460 & -28.4466 & -29.1449 & -29.1446 & -29.1338 \\
$\mathrm{~B}$ & -49.0586 & -48.1272 & -49.0567 & -49.0637 & -49.0476 \\
$\mathrm{C}$ & -75.3800 & -74.2237 & -75.3778 & -75.3871 & -75.3681 \\
$\mathrm{~N}$ & -108.8091 & -107.4184 & -108.8068 & -108.8063 & -108.7846 \\
$\mathrm{O}$ & -149.6273 & -147.9836 & -149.6241 & -149.6357 & -149.6081 \\
$\mathrm{~F}$ & -198.8217 & -196.9479 & -198.8184 & -198.8324 & -198.8013 \\
$\mathrm{Ne}$ & -257.0942 & -254.9815 & -257.0908 & -257.0898 & -257.0553 \\
$\mathrm{Na}$ & -323.7180 & -321.2885 & -323.7132 & -323.7119 & -323.6721 \\
$\mathrm{Mg}$ & -399.2292 & -396.4976 & -399.2232 & -399.2212 & -399.1826 \\
$\mathrm{Al}$ & -483.7536 & -480.7121 & -483.7466 & -483.7497 & -483.7108 \\
$\mathrm{Si}$ & -577.7090 & -574.3641 & -577.7014 & -577.7058 & -577.6659 \\
$\mathrm{P}$ & -681.4386 & -677.7771 & -681.4300 & -681.4275 & -681.3861 \\
$\mathrm{~S}$ & -795.0126 & -791.0380 & -795.0032 & -795.0088 & -794.9659 \\
$\mathrm{Cl}$ & -918.9652 & -914.6869 & -918.9652 & -918.9617 & -918.9174 \\
$\mathrm{Ar}$ & -1053.6350 & -1049.0349 & -1053.6244 & -1053.6207 & -1053.5754 \\
\hline \hline
\end{tabular}

${ }^{\mathrm{a}}$ Reference [26].

TABLE III. Total energies for atoms from $\mathrm{K}$ to $\mathrm{Kr}$, which are calculated from the HF, xLSDA, OEP, KLI, and present schemes. The HF and OEP total energies are taken from Ref. [25]. All values are in Rydberg units.

\begin{tabular}{lccccc}
\hline \hline & HF & xLSDA & OEP & KLI & Present \\
\hline $\mathrm{K}$ & -1198.3298 & -1193.4228 & -1198.3182 & -1198.3141 & -1198.2652 \\
$\mathrm{Ca}$ & -1353.5164 & -1348.3202 & -1353.5038 & -1353.4994 & -1353.4505 \\
$\mathrm{Sc}$ & -1519.4718 & -1514.0166 & -1519.4554 & -1519.4092 & -1519.4226 \\
$\mathrm{Ti}$ & -1696.8132 & -1691.0612 & -1696.7948 & -1696.7874 & -1696.7493 \\
$\mathrm{~V}$ & -1885.7712 & -1879.7465 & -1885.7520 & -1885.7429 & -1885.7053 \\
$\mathrm{Cr}$ & -2086.7136 & -2080.5463 & -2086.6914 & -2086.6835 & -2086.6330 \\
$\mathrm{Mn}$ & -2299.7396 & -2293.1656 & -2299.7200 & -2299.7129 & -2299.6566 \\
$\mathrm{Fe}$ & -2524.9000 & -2518.0766 & -2524.8760 & -2524.7344 & -2524.8407 \\
$\mathrm{Co}$ & -2762.8372 & -2755.7211 & -2762.8112 & -2762.7902 & -2762.7566 \\
$\mathrm{Ni}$ & -3013.7464 & -3006.3762 & -3013.7196 & $-3013.7120^{\mathrm{a}}$ & -3013.6628 \\
$\mathrm{Cu}$ & -3277.9284 & -3270.4781 & -3277.9046 & $-3277.8962^{\mathrm{a}}$ & -3277.8189 \\
$\mathrm{Zn}$ & -3555.6962 & -3547.8199 & -3555.6688 & -3555.6634 & -3555.5907 \\
$\mathrm{Ga}$ & -3846.5224 & -3838.1902 & -3846.4974 & -3846.4966 & -3846.4250 \\
$\mathrm{Ge}$ & -4150.7206 & -4141.9620 & -4150.6966 & -4150.6982 & -4150.6245 \\
$\mathrm{As}$ & -4468.4798 & -4459.2948 & -4468.4562 & -4468.4498 & -4468.3765 \\
$\mathrm{Se}$ & -4799.7382 & -4790.1519 & -4799.7146 & -4799.7156 & -4799.6435 \\
$\mathrm{Br}$ & -5144.8836 & -5134.9093 & -5144.8600 & -5144.8635 & -5144.7885 \\
$\mathrm{Kr}$ & -5504.1100 & -5493.7323 & -5504.0860 & -5504.0790 & -5504.0065 \\
\hline \hline $\mathrm{Re} e r$ & {$[25$} & & & &
\end{tabular}

${ }^{\mathrm{a}}$ Reference [25]. 
TABLE IV. Total energies for atoms from $\mathrm{Rb}$ to $\mathrm{Xe}$, which are calculated from the HF, xLSDA, OEP, KLI, and present schemes. The HF and OEP total energies are taken from Ref. [25]. All values are in Rydberg units.

\begin{tabular}{lccccc}
\hline \hline & HF & xLSDA & OEP & KLI & Present \\
\hline $\mathrm{Rb}$ & -5876.7152 & -5865.9670 & -5876.6910 & -5876.6851 & -5876.6114 \\
$\mathrm{Sr}$ & -6263.0914 & -6251.9963 & -6263.0668 & -6263.0595 & -6262.9886 \\
$\mathrm{Y}$ & -6663.3692 & -6651.9424 & -6663.3420 & -6663.3283 & -6663.2801 \\
$\mathrm{Zr}$ & -7078.0234 & -7066.2069 & -7077.9940 & -7077.9887 & -7077.8932 \\
$\mathrm{Nb}$ & -7507.2012 & -7495.1268 & -7507.1710 & -7507.1732 & -7507.1072 \\
$\mathrm{Mo}$ & -7951.1060 & -7938.6642 & -7951.0742 & -7951.0665 & -7950.9898 \\
$\mathrm{Tc}$ & -8409.5898 & -8396.7661 & -8409.5586 & $-8409.5482^{\mathrm{a}}$ & -8409.4721 \\
$\mathrm{Ru}$ & -8883.0818 & -8869.9024 & -8883.0490 & $-8883.0380^{\mathrm{a}}$ & -8882.9695 \\
$\mathrm{Rh}$ & -9371.7644 & -9358.2350 & -9371.7312 & -9371.7297 & -9371.6473 \\
$\mathrm{Pd}$ & -9875.8420 & -9862.0202 & -9875.8120 & -9875.8045 & -9875.7061 \\
$\mathrm{Ag}$ & -10395.3978 & -10381.1564 & -10395.3630 & -10395.3509 & -10395.2592 \\
$\mathrm{Cd}$ & -10930.2662 & -10915.6438 & -10930.2288 & $-10930.2168^{\mathrm{a}}$ & -10930.1326 \\
$\mathrm{In}$ & -11480.3388 & -11465.2983 & -11480.3028 & $-11480.2910^{\mathrm{a}}$ & -11480.2129 \\
$\mathrm{Sn}$ & -12045.8650 & -12030.4256 & -12045.8298 & $-12045.8184^{\mathrm{a}}$ & -12045.7411 \\
$\mathrm{Sb}$ & -12626.9740 & -12611.1314 & -12626.9394 & $-12626.9278^{\mathrm{a}}$ & -12626.8436 \\
$\mathrm{Te}$ & -13223.5712 & -13207.3537 & -13223.5366 & $-13223.5250^{\mathrm{a}}$ & -13223.4489 \\
$\mathrm{I}$ & -13835.9628 & -13819.3802 & -13835.9284 & $-13835.9164^{\mathrm{a}}$ & -13835.8415 \\
$\mathrm{Xe}$ & -14464.2774 & -14447.3146 & -14464.2420 & $-14464.2300^{\mathrm{a}}$ & -14464.1478 \\
\hline $\mathrm{a} \mathrm{Refen}$ & {$[25]$} & & & &
\end{tabular}

${ }^{\mathrm{a}}$ Reference [25].

$$
\frac{e^{2}}{2 \rho_{\sigma}(\mathbf{r})} \sum_{i=1}^{N_{\sigma}}\left|\psi_{i \sigma}(\mathbf{r})\right|^{2}\left[u_{x i \sigma}(\mathbf{r})+u_{x i \sigma}^{*}(\mathbf{r})\right]=\int d \mathbf{r}^{\prime} \frac{\rho_{x \sigma}\left(\mathbf{r}, \mathbf{r}^{\prime}\right)}{\left|\mathbf{r}-\mathbf{r}^{\prime}\right|}
$$

This indicates that the difference between the calculation time for the constant terms and that for $\beta_{x}$ causes the improvements mentioned above. Namely, the numerical calculation of the constant terms in the KLI approximation takes

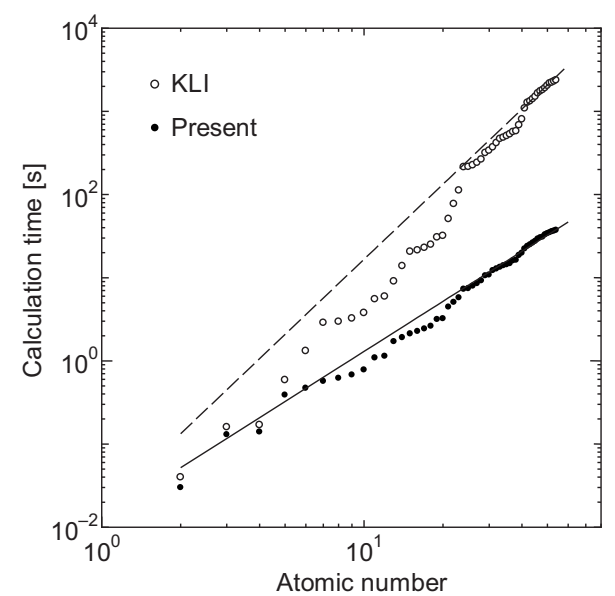

FIG. 2. Calculation time required for one iteration for the KLI approximation and the present scheme. The solid and dashed lines, which are guides for the eyes, are proportional to the square of the atomic number and to the cube of the atomic number, respectively. much time, while the calculation of the constant $\beta_{x}$ is not so heavy even if the atomic number increases.

\section{Renormalization factor}

In Fig. 3, we show the renormalization factor $\beta_{x}$ for atoms from $\mathrm{He}$ to $\mathrm{Xe}$. For the $\mathrm{He}$ atom, $\beta_{x}$ is unity. As the atomic number increases, $\beta_{x}$ decreases and approaches 0.7 . This means that the sign of the second term of Eq. (4) is opposite

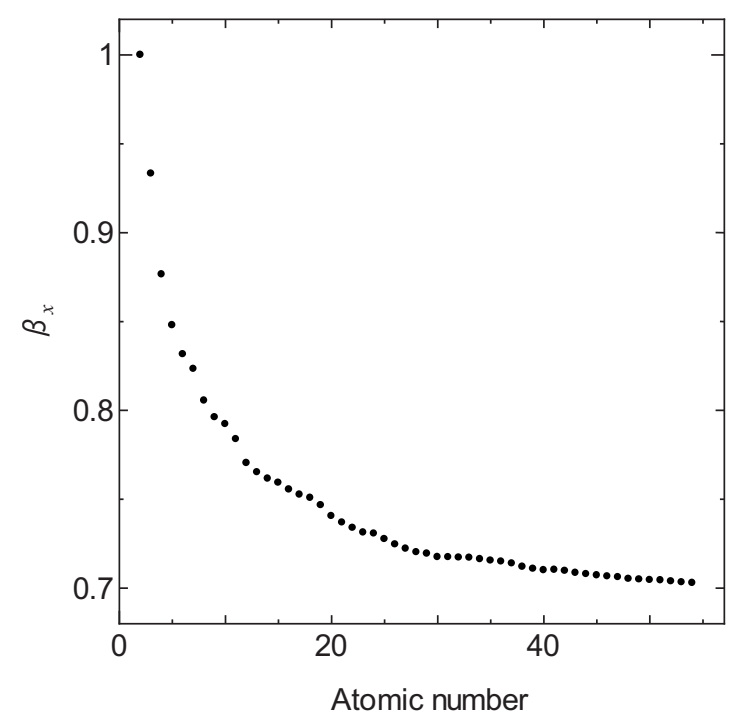

FIG. 3. The renormalization factor $\beta_{x}$ are shown as a function of the atomic number. 
to that of the first term and its magnitude is not negligibly small. These properties are consistent with the results of the direct calculations of the first and second terms $[19,20]$. We can also confirm that the value of $\beta_{x}$ for Be atom is almost the same as one evaluated near the nucleus in the $\mathrm{x}$-only OEP calculation [19].

\section{CONCLUSION}

We propose a scheme for calculating the orbitaldependent exchange-correlation potential by introducing the renormalization constant factor. The effect of the functional derivative of the exchange-correlation hole with respect to the electron density is renormalized into this factor. Its value is determined by requiring that the exchange-correlation potential holds the virial theorem of the DFT. This method is essentially different from the OEP method.

In order to confirm the advantage of this scheme, we perform the $\mathrm{x}$-only calculations for atoms from $\mathrm{He}$ to Xe. The accuracy of the present scheme is almost the same as that of the KLI approximation. As for the calculation speed, the present scheme becomes dramatically faster than the KLI approximation as the atomic number increases. It is concluded that the present scheme is easier to handle computationally than the OEP method. The important point is to reduce the computational tasks without losing numerical accuracy. Judging from the results for atoms, the present scheme seems to be effective in application to solids.

\section{ACKNOWLEDGMENTS}

We acknowledge Professor Tamio Oguchi and Dr. Tatsuya Shishidou for valuable discussions. This work was partially supported by a Grant-in-Aid for Scientific Research (No. 19540399) and for Scientific Research in Priority Areas (No. 17064006) of The Ministry of Education, Culture, Sports, Science, and Technology Japan.
[1] P. Hohenberg and W. Kohn, Phys. Rev. 136, B864 (1964).

[2] W. Kohn and L. J. Sham, Phys. Rev. 140, A1133 (1965).

[3] For instance, see R. M. Dreizler and E. K. U. Gross, Density Functional Theory (Springer, Berlin, 1990); R. G. Parr and W. Yang, Density Functional Theory of Atoms and Molecules (Oxford University Press, Oxford, 1989).

[4] R. T. Sharp and G. K. Horton, Phys. Rev. 90, 317 (1953).

[5] J. D. Talman and W. F. Shadwick, Phys. Rev. A 14, 36 (1976).

[6] S. Kümmel and L. Kronik, Rev. Mod. Phys. 80, 3 (2008).

[7] J. B. Krieger, Y. Li, and G. J. Iafrate, Phys. Lett. A 146, 256 (1990); Phys. Rev. A 45, 101 (1992); 46, 5453 (1992).

[8] O. Gritsenko, R. van Leeuwen, E. van Lenthe, and E. J. Baerends, Phys. Rev. A 51, 1944 (1995).

[9] L. Fritsche and J. Yuan, Phys. Rev. A 57, 3425 (1998).

[10] S. Ivanov, S. Hirata, and R. J. Bartlett, Phys. Rev. Lett. 83, 5455 (1999).

[11] A. Görling, Phys. Rev. Lett. 83, 5459 (1999).

[12] R. Colle and R. K. Nesbet, J. Phys. B 34, 2475 (2001).

[13] O. V. Gritsenko and E. J. Baerends, Phys. Rev. A 64, 042506 (2001).

[14] W. Yang and Q. Wu, Phys. Rev. Lett. 89, 143002 (2002).

[15] S. Kümmel and J. P. Perdew, Phys. Rev. Lett. 90, 043004 (2003).

[16] V. N. Staroverov, G. E. Scuseria, and E. R. Davidson, J. Chem.
Phys. 124, 141103 (2006).

[17] A. D. Becke and E. R. Johnson, J. Chem. Phys. 124, 221101 (2006).

[18] M. Levy and J. P. Perdew, Phys. Rev. A 32, 2010 (1985).

[19] R. van Leeuwen, O. V. Gritsenko, and E. J. Baerends, Z. Phys. D: At., Mol. Clusters 33, 229 (1995).

[20] J. A. Alonso and N. A. Cordero, in Recent Developments and Applications of Modern Density Functional Theory (Elsevier, Amsterdam, 1996), Chap. 7, p. 239.

[21] By the coordinate scaling of electrons, $\rho(\mathbf{r})$ is transformed into $\rho_{\lambda}(\mathbf{r})\left[=\lambda^{3} \rho(\lambda \mathbf{r})\right]$, where $\lambda$ is the scale factor. As shown in Ref. [18], the exact relation $E_{x}\left[\rho_{\lambda}\right]=\lambda E_{x}[\rho]$ holds. Acting with the operator $\lim _{\lambda \rightarrow 1}(\partial / \partial \lambda)$ on both sides of this relation, and integrating by parts, we arrive at Eq. (11).

[22] For instance, see R. M. Dreizler and E. K. U. Gross, Density Functional Theory (Springer, Berlin, 1990), Chap. 4, p. 68.

[23] A. D. Becke, J. Chem. Phys. 84, 4524 (1986).

[24] E. Engel and S. H. Vosko, Phys. Rev. A 47, 2800 (1993).

[25] Y. Li, J. B. Krieger, and G. J. Iafrate, Phys. Rev. A 47, 165 (1993).

[26] T. Grabo, T. Kreibich, S. Kurth, and E. K. U. Gross, in Strong Coulomb Correlations in Electronic Structure Calculations: Beyond the Local Density Approximation, edited by V. I. Anisimov (Gordon and Breach, Amsterdam, 2000), p. 203. 